\title{
Ectopic development of skeletal muscle induced by subcutaneous transplant of rat satellite cells
}

\author{
M.G. Fukushima ${ }^{1}$, \\ I. Furlan ${ }^{1}$, T. Chiavegatti ${ }^{1}$, \\ B.H. Kiyomoto ${ }^{2}$ \\ and R.O. Godinho ${ }^{1}$
}

\author{
Departamentos de ${ }^{1}$ Farmacologia and ${ }^{2}$ Neurologia e Neurocirurgia, \\ Universidade Federal de São Paulo, Escola Paulista de Medicina, \\ São Paulo, SP, Brasil
}

\author{
Correspondence \\ R.O. Godinho \\ Departamento de Farmacologia \\ EPM, UNIFESP \\ Rua 3 de maio, 100 \\ 04044-020 São Paulo, SP \\ Brasil \\ Fax: +55-11-5576-4499 \\ E-mail: godinho@farm.epm.br \\ Research supported by FAPESP \\ (No. 01/01417-5). M.G. Fukushima \\ was recipient of a PIBIC/CNPq \\ fellowship. T. Chiavegatti and \\ I. Furlan were recipients of \\ CNPq and FAPESP MS fellowships, \\ respectively.
}

Received June 21, 2004

Accepted November 23, 2004

$\ldots \ldots \ldots \ldots \ldots \ldots \ldots \ldots$

\begin{abstract}
The present study analyzes the ectopic development of the rat skeletal muscle originated from transplanted satellite cells. Satellite cells $\left(10^{6}\right.$ cells) obtained from hindlimb muscles of newborn female 2BAW Wistar rats were injected subcutaneously into the dorsal area of adult male rats. After 3, 7, and 14 days, the transplanted tissues $(\mathrm{N}=4-5)$ were processed for histochemical analysis of peripheral nerves, inactive X-chromosome and acetylcholinesterase. Nicotinic acetylcholine receptors (nAChRs) were also labeled with tetramethylrhodaminelabeled $\alpha$-bungarotoxin. The development of ectopic muscles was successful in $86 \%$ of the implantation sites. By day 3 , the transplanted cells were organized as multinucleated fibers containing multiple clusters of nAChRs $(\mathrm{N}=2-4)$, resembling those from non-innervated cultured skeletal muscle fibers. After 7 days, the transplanted cells appeared as a highly vascularized tissue formed by bundles of fibers containing peripheral nuclei. The presence of $\mathrm{X}$ chromatin body indicated that subcutaneously developed fibers originated from female donor satellite cells. Differently from the extensor digitorum longus muscle of adult male rat $(87.9 \pm 1.0 \mu \mathrm{m} ; \mathrm{N}=213)$, the diameter of ectopic fibers $(59.1 \mu \mathrm{m} ; \mathrm{N}=213)$ did not obey a Gaussian distribution and had a higher coefficient of variation. After 7 and 14 days, the organization of the nAChR clusters was similar to that of clusters from adult innervated extensor digitorum longus muscle. These findings indicate the histocompatibility of rats from $2 \mathrm{BAW}$ colony and that satellite cells transplanted into the subcutaneous space of adult animals are able to develop and fuse to form differentiated skeletal muscle fibers.
\end{abstract}

\section{Introduction}

The development of skeletal muscle fibers initiates during embryogenesis, when mononucleated myoblasts derived from pluripotent mesodermic cells fuse to form multinucleated myotubes. Subsequently, these fusiform cells differentiate into skeletal muscle fibers with
Key words

- Skeletal muscle

- Satellite cells

- Stem cells

- Transplantation

- Myogenesis

- 2BAW rats peripheral nuclei, a mature contractile apparatus and compartmentalized expression of synaptic proteins such as nicotinic acetylcholine receptors (nAChRs) and acetylcholinesterase (AChE) (1). Beyond this stage, mitosis and DNA synthesis arrest almost completely and the adult muscle fibers lack the ability to proliferate. Upon injury, however, quiescent myo- 
genic precursor cells (satellite cells) located under the basal lamina of individual postnatal and adult myofibers can be activated, leading to complete regeneration of skeletal muscle. Once activated, these cells proliferate and fuse to form new myofibers morphologically and functionally indistinguishable from undamaged ones $(2,3)$. In fact, recent studies indicate that satellite cells represent a unique population of progenitor cells committed to the myogenic lineage and derived from multipotent muscle-derived stem cells (4).

The demonstration that intramuscular injection of myoblasts induces fusion between host and donor myoblasts/satellite cells in skeletal muscle grafts $(5,6)$ initiated a series of studies focusing on the transplantation of myogenic cells as a potential therapeutic strategy for treatment of skeletal muscle disorders, including Duchenne muscular dystrophy. More recently, the therapeutic benefits of transplantation of skeletal muscle precursors (myoblasts/satellite cells) extended to treatment of myocardial diseases, such as infarction and end-stage heart failure (for a review, see Ref. 7). In different animal species, it has been reported that injection of myoblasts/satellite cells into the heart muscle improves cardiac contraction (8-10). Interestingly, at this site, the implanted cells acquire some of the contractile properties of cardiomyocytes $(8,11)$.

Assuming that differentiation of multipotent cells occurs in response to signals provided by their host tissue (12), in the present study we investigated whether freshly isolated myogenic precursor cells from newborn 2BAW rats were able to differentiate into skeletal muscle fibers in the subcutaneous region, an environment free from skeletal muscle cells.

\section{Material and Methods}

The experiments were conducted on adult male (3 months old) and newborn female Wistar 2BAW rats. The adult rats were kept on 12-h light-dark cycles with free access to tap water and food. All protocols were in accordance with the Guide for the Care and Use of Laboratory Animals (National Institutes of Health, 1986).

\section{Isolation and implantation of satellite cells}

Satellite cells were obtained from hindlimb muscles of newborn female Wistar rats. Briefly, the animals were killed under $\mathrm{CO}_{2}$ anesthesia, the muscles were removed and the cells dissociated for $3 \mathrm{~h}$ in Hanks' balanced salt solution (HBSS), $\mathrm{pH} 7.4$, containing collagenase type IA $(200 \mathrm{U} / \mathrm{ml})$ as described by da Costa et al. (13). After mechanical dissociation, the cell suspension was centrifuged at $500 \mathrm{~g}$ for $5 \mathrm{~min}$ and the resulting pellet was resuspended in Dulbecco's Modified Eagle's Medium (DMEM, Gibco-BRL, Life Technologies, Grand Island, NY, USA) containing $40 \mathrm{mg} / 1$ gentamicin. To avoid fibroblast contamination, the cells were preincubated in culture flasks for $30 \mathrm{~min}$ at $37^{\circ} \mathrm{C}$ in a humidified atmosphere of $90 \%$ air and $10 \% \mathrm{CO}_{2}$. Non-attached mononucleated satellite cells were rinsed three times with HBSS and injected subcutaneously $\left(10^{6}\right.$ cells $\left./ 200 \mu \mathrm{l}\right)$ into the dorsal area of adult male Wistar rats using a $0.5 \mathrm{ml}$ syringe with a 26 -gauge needle.

In another set of experiments, $10^{6}$ satellite cells were diluted in $2 \mathrm{ml}$ DMEM supplemented with $15 \%$ fetal calf serum, seeded onto $35-\mathrm{mm}$ collagen-coated culture dishes and maintained at $37^{\circ} \mathrm{C}$ in a humidified atmosphere of $90 \%$ air and $10 \% \mathrm{CO}_{2}$ (14). On the third day and every other day thereafter the medium was replaced with DMEM supplemented with $10 \%$ horse serum (HS) and $2 \%$ fetal calf serum. On day 7 , myotubes were multinucleated and contracting cells indicated an appropriate maturation of the muscle fibers in vitro.

\section{Processing transplanted tissue}

After 3,7 or 14 days the recipient rats 
were sacrificed by section of the abdominal aorta under ether anesthesia. The skin from the dorsal area was removed and transferred to a Petri dish containing phosphate-buffered saline (PBS), $\mathrm{pH}$ 7.4. The transplanted tissues were dissected, weighed, covered with tissue freezing medium (Triangle Biomedical Sciences, Durham, NC, USA) and immediately frozen in liquid nitrogen. The tissues were sectioned on a cryostat at a thickness of 8-10 $\mu \mathrm{m}$, mounted on slides and kept frozen at $-80^{\circ} \mathrm{C}$ until the time for histological analysis. The extensor digitorum longus (EDL) muscle from adult rats was used as control.

\section{Determination of fiber diameter}

The sections were stained with hematoxylin and eosin, dehydrated by successive immersions in $70 \%, 95 \%$ and absolute ethanol, and dried. The slides were then immersed in xylene, mounted with Entellan ${ }^{\circledR}$ neu (Merck, Darmstadt, Germany) and examined under a light microscope. At least three sections from each implant were photographed with 40 and $60 \mathrm{X}$ objectives and the diameter of up to 213 fibers was determined using public domain NIH-Image 1.61 software. Frequency histograms of the fiber diameter were constructed and compared to that obtained from the EDL muscle of adult male rats.

\section{Acetylcholinesterase staining}

Sections were fixed in 2\% paraformaldehyde for $1 \mathrm{~h}$, rinsed with PBS and incubated in $100 \mathrm{mM}$ acetate buffer, $\mathrm{pH}$ 6.0, containing $2 \mathrm{mM}$ acetylthiocholine iodide, $10 \mathrm{mM}$ sodium citrate, $3 \mathrm{mM}$ copper sulfate, and 0.5 $\mathrm{mM}$ potassium ferricyanide at $25^{\circ} \mathrm{C}$, according to the method of Karnovsky and Roots (15). After 1-2 h, the sections were rinsed in PBS, counterstained with hematoxylin and mounted with 9:1 glycerin/PBS solution, $\mathrm{pH}$ 7.4.

\section{Peripheral nerve staining}

In order to analyze the possible innervation of muscle fibers, the peripheral neurons were stained with the Bielschowsky silver stain modified by Mirra et al. (16). After AChE staining, tissue sections were incubated with $20 \%$ silver nitrate solution for 15 $\min$ at $37^{\circ} \mathrm{C}$. The slides were then rinsed three times with water and incubated with ammoniacal silver solution at $37^{\circ} \mathrm{C}$. After 10 min, the sections were incubated with developer solution containing $0.5 \%$ formaldehyde, $50 \mu \mathrm{M} \mathrm{HNO}_{3}, 160 \mu \mathrm{M}$ citric acid, and $0.2 \%$ ammonium hydroxide for 1-2 min, and rinsed successively with $0.2 \%$ ammonium hydroxide and distilled water. The reaction was stopped with 5\% sodium thiosulfate and the slides were finally washed again in water, dehydrated and mounted with Entellan ${ }^{\circledR}$ neu.

\section{Nicotinic acetylcholine receptor staining}

Sections were rinsed for $10 \mathrm{~min}$ with DMEM containing 10\% HS (DMEM-HS) and incubated with tetramethylrhodaminelabeled $\alpha$-bungarotoxin (TRICT- $\alpha$-BTX; 1 $\mu \mathrm{g} / \mathrm{ml}$ in PBS; Molecular Probes, Eugene, OR, USA) and Hoescht dye $33258(0.5 \mu \mathrm{g} /$ $\mathrm{ml}$; Molecular Probes) at $25^{\circ} \mathrm{C}$, in order to stain the nAChRs and nuclei, respectively. After $1 \mathrm{~h}$, the sections were washed three times with DMEM-HS and once with PBS and covered with an anti-quenching gel mounting solution (Biomeda Corp., Foster City, CA, USA).

\section{Inactive $X$ chromosome staining}

To identify the inactive $\mathrm{X}$ chromosome from female rat donors the sections from implanted satellite cells were stained with cresyl-violet. The sex chromatin body appeared as deeply stained structures in a subnuclear membrane location. Briefly, the sections were fixed in methanol/acetic acid solution 3:1 (v/v). After $30 \mathrm{~min}$, the samples 
were immersed in $5 \mathrm{M} \mathrm{HCl}$ for $10 \mathrm{~min}$ for acid hydrolysis and rinsed in distilled water. The samples were then incubated in $0.1 \%$ cresyl violet solution for $30 \mathrm{~min}$, rinsed five times with water and mounted with Entellan ${ }^{\circledR}$ neu.

\section{Statistical analysis}

Data are reported as the means \pm SEM of at least three implants. The distribution of fiber diameter was submitted to a normality test using GraphPad Prism v.3 software

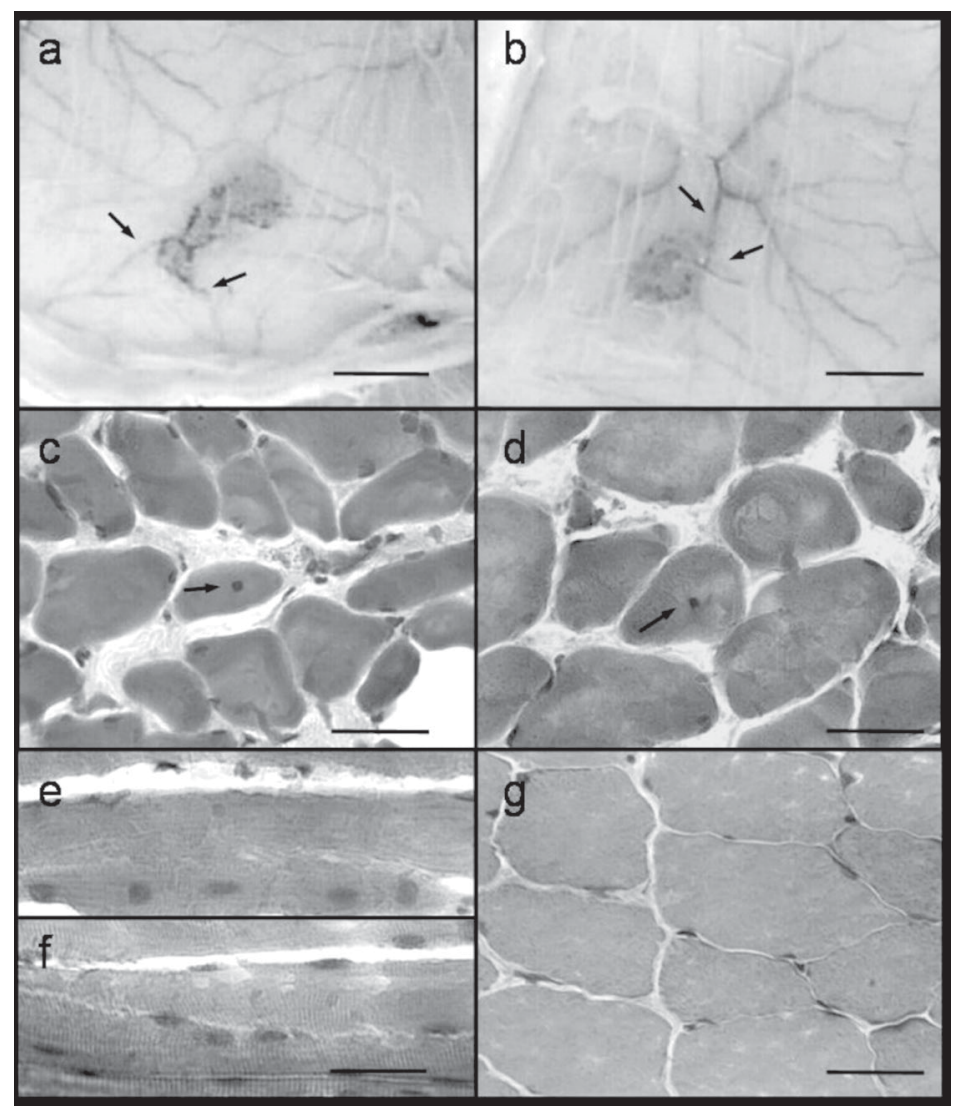

Figure 1. Ectopic development of skeletal muscle induced by subcutaneous transplantation of rat satellite cells. Satellite cells $\left(10^{6}\right.$ cells $)$ obtained from hindlimb muscles of newborn female rats were injected subcutaneously into the dorsal region of adult male rats. After 7 days, the transplants were removed, processed and stained with hematoxylin and eosin (HE) $(a, b$; Bars, $1 \mathrm{~cm})$. A tissue mass formed under the skin of adult male rat after transplantation of satellite cells. Cross-sections $(c, d, g)$ or longitudinal sections $(e, f)$ of transplanted tissue (c-f) or extensor digitorum longus muscle from an adult male rat (g), stained with $\mathrm{HE}$ (Bars, $50 \mu \mathrm{m})$. The arrows indicate the presence of vascular formation $(a, b)$ and the central nuclei $(c, d)$.
(GraphPad Software, Inc., San Diego, CA, USA). Differences between means were analyzed by the Student $t$-test, with the level of significance set at $\mathrm{P}<0.05$.

\section{Results}

Figure 1a,b shows the subcutaneous layer from host rats 7 days after satellite cell transplantation. The transplanted cells appear as a highly vascularized and well-defined tissue weighing $23.4 \pm 2.8 \mathrm{mg}(\mathrm{N}=5)$. HE staining of transplant sections showed multi-

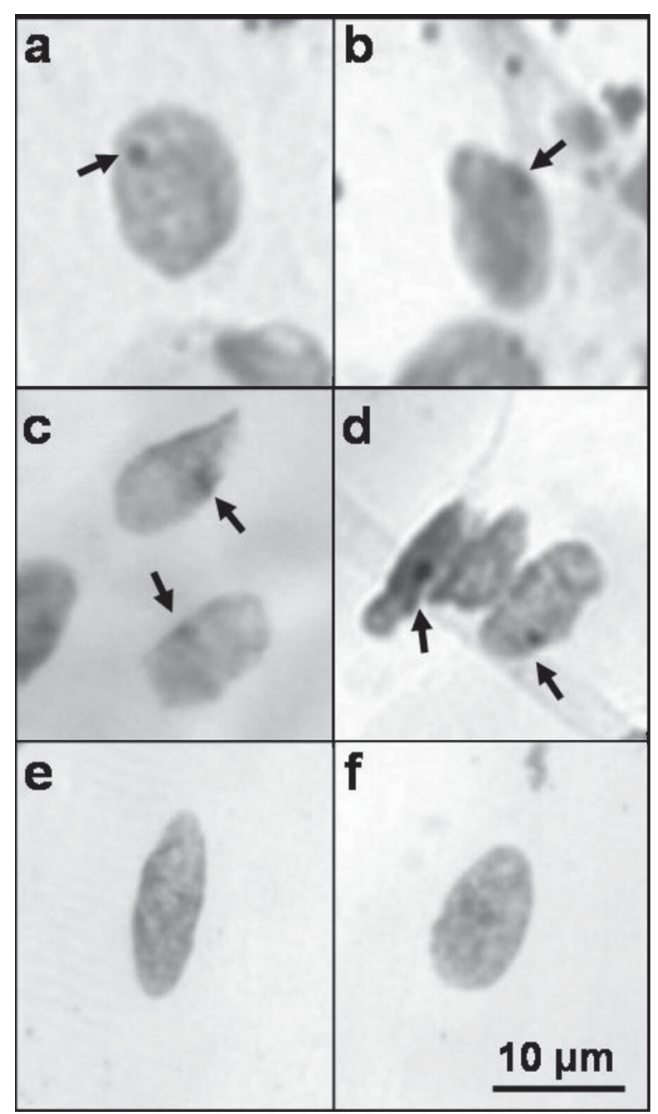

Figure 2. Inactive $X$ chromosome of the skeletal muscle cells. In order to identify the origin of ectopically developed muscle fibers, sections obtained 7 days after transplantation of female satellite cells were stained with cresyl violet and compared to extensor digitorum longus muscle (EDL) sections from adult female and male rats. Nuclei containing an inactive $X$ chromosome (indicated by the arrows) were observed in both allografts $(a, b)$ and female EDL $(c, d)$, but not in male EDL $(e, f)$. 
nucleated cells (Figure 1c-f) that resemble those from adult skeletal muscle (EDL, Figure 1g). Although most of the ectopic fibers displayed characteristics of mature muscle fibers, such as cross-striations and peripheral nuclei, myotubes with centrally located nuclei were also found (Figure 1c,d, arrows), indicating the presence of fibers in intermediate stages of differentiation.

The presence of $\mathrm{X}$ inactive chromatin, identified as a dark stained body in close association with the nuclear membrane, ensured that subcutaneous muscle fibers were derived from female donor satellite cells (Figure 2a,b). The $\mathrm{X}$ chromosome positive nuclei were also observed in EDL from adult female rats (Figure 2c,d) but not from male rats (Figure 2e,f).

The transplanted tissue also exhibited distinct features when compared to the EDL sections, such as a higher amount of connective tissue (Figure 1c,d) and high variability of fiber sizes. Figure 3 shows the frequency histogram of fiber diameters 7 days after cell transplantation in comparison to the EDL from adult male rats. The mean diameter of fibers from transplants was smaller (59.1 $\mu \mathrm{m} ; \mathrm{N}=213$ ) than that of fibers from EDL
$(87.9 \pm 1.0 \mu \mathrm{m}, \mathrm{N}=213)$ and did not obey a normal distribution. By day 7, the coefficient of variation of fiber diameters was $33.6 \%$ whereas that from adult EDL fiber was only $16 \%$.

In order to analyze the developmental expression of nAChRs, receptors were stained with TRICT- $\alpha$-BTX. Three days after transplantation, ectopic fibers expressed multiple clusters of nAChRs (Figure 4a) that resembled those from non-innervated cultured skeletal muscle fibers (Figure 4b). By days 7 and 14, however, the nAChRs were distributed in a single cluster per fiber (Figure 5a-d), an arrangement similar to that observed in innervated EDL from adult rats (Figure 5e,f).

At this stage, the transplanted fibers also exhibited localized expression of AChE (data not shown). Since synaptic contact is responsible for the organization of nAChRs and AChE clusters in vivo, we investigated the possible innervation of ectopic skeletal muscle fibers. As shown in Figure 6a-d, black-stained axons and intramuscular nerve branches were detected after silver impregnation, indicating the innervation of ectopic muscle fibers.

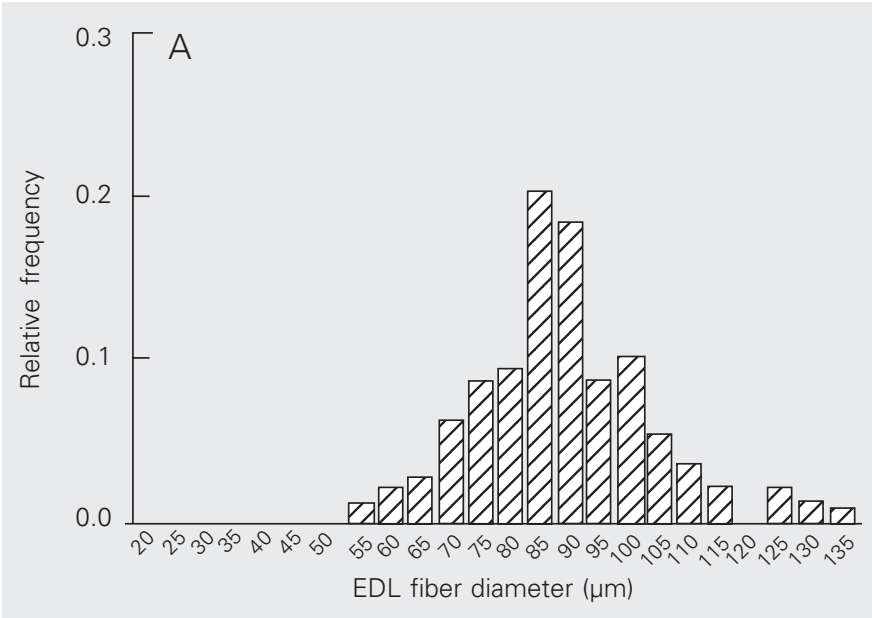

Figure 3. Frequency histograms of skeletal muscle fiber diameter. Sections obtained from adult male rat extensor digitorum longus (EDL) muscle (A) or from ectopic muscle 7 days after transplantation (B) were stained with hematoxylin and eosin and photographed with $40 \mathrm{X}$ objectives and the fiber diameter was measured using public domain $\mathrm{NIH}$-Image 1.61 software.

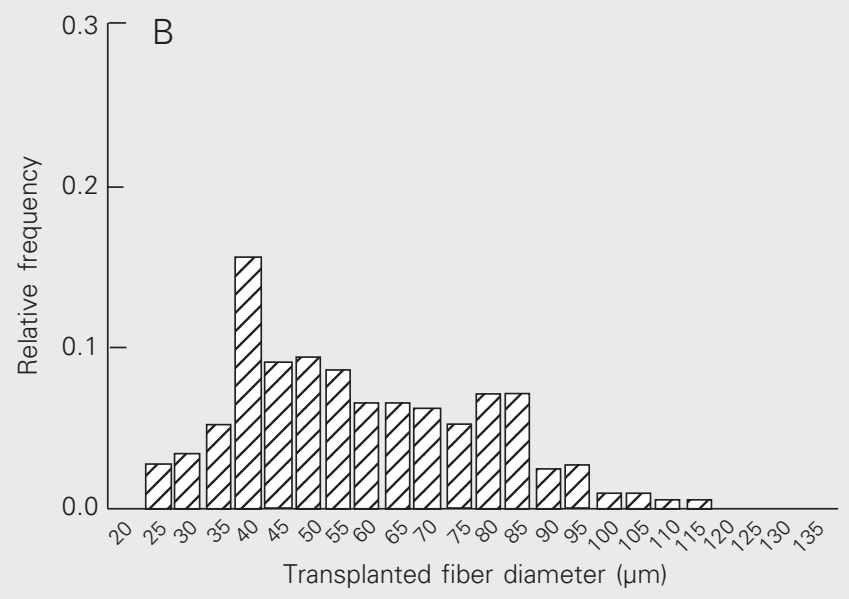

Braz J Med Biol Res 38(3) 2005 
Figure 4. Nicotinic acetylcholine receptor clusters in ectopic muscle and in cultured muscle fibers. Tissue sections from 3-day ectopic skeletal muscle fibers (a) or from cultured rat skeletal muscle fibers (b) were labeled with tetramethylrhodamine-labeled $\alpha$ bungarotoxin

Figure 5. Nicotinic acetylcholine receptor (nAChR) clusters expressed in ectopic muscle and in the extensor digitorum longus (EDL) endplate from adult male rats. Tissue sections from 7-day $(a, b)$ or 14-day $(c, d)$ ectopic skeletal muscle fibers were labeled with tetramethylrhodamine-labeled $\alpha$-bungarotoxin and the distribution of nAChRs was compared to that observed in the neuromuscular junction of EDL from an adult male rat $(e, f)$.

Figure 6. Detection of a peripheral nerve at the site of ectopic skeletal muscle fibers. Fourteen days after transplantation of satellite cells, the allografts were submitted to peripheral nerve staining (stained in black, a-d).
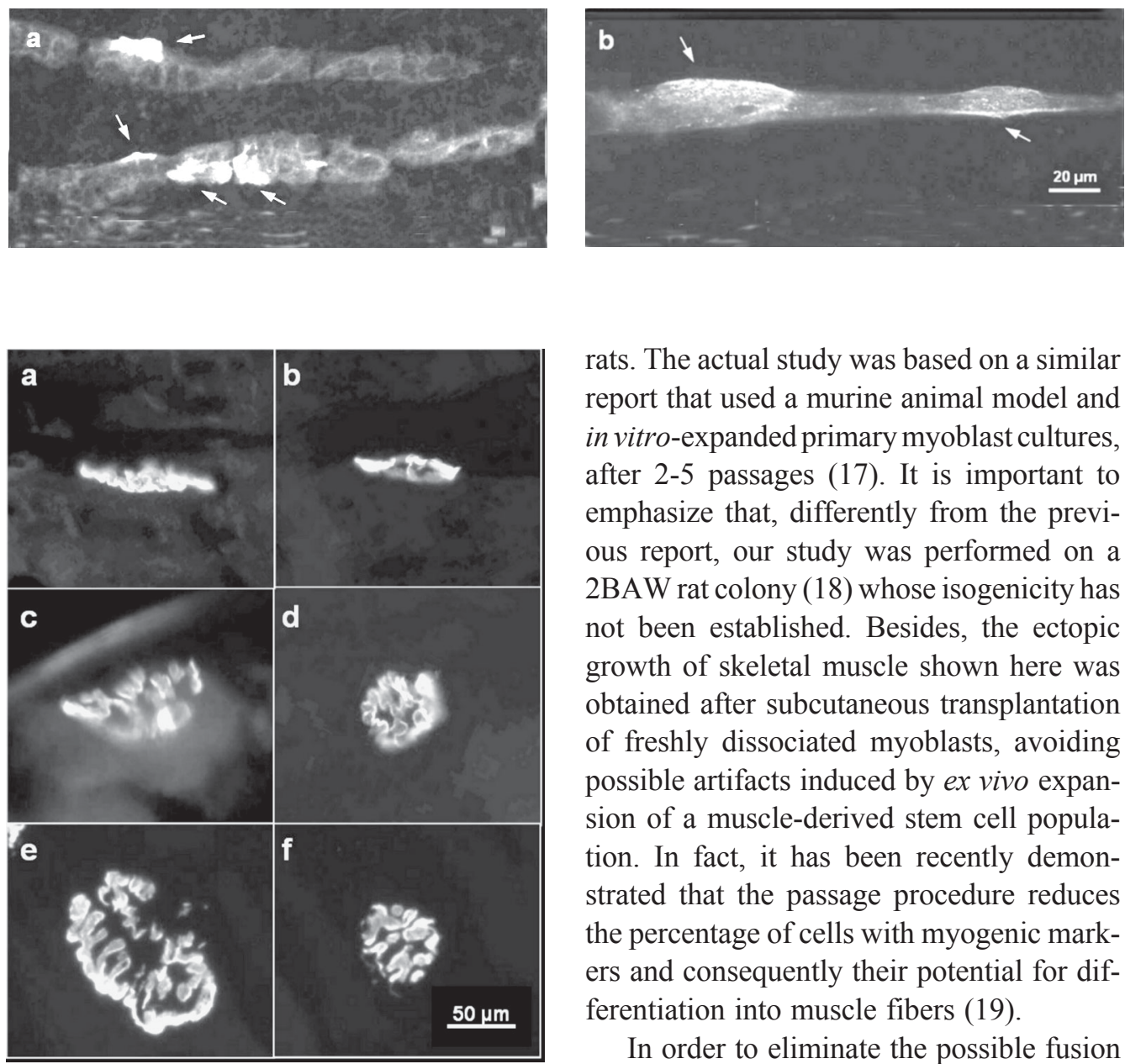

rats. The actual study was based on a similar report that used a murine animal model and in vitro-expanded primary myoblast cultures, after 2-5 passages (17). It is important to emphasize that, differently from the previous report, our study was performed on a 2BAW rat colony (18) whose isogenicity has not been established. Besides, the ectopic growth of skeletal muscle shown here was obtained after subcutaneous transplantation of freshly dissociated myoblasts, avoiding possible artifacts induced by ex vivo expansion of a muscle-derived stem cell population. In fact, it has been recently demonstrated that the passage procedure reduces the percentage of cells with myogenic markers and consequently their potential for differentiation into muscle fibers (19).

In order to eliminate the possible fusion of transplanted satellite cells with host muscle fibers or satellite cells, which could modify the development of implants, the subcutaneous space, a skeletal muscle-free environment, was used as the recipient location. In addition, the detection of an inactive $\mathrm{X}$ chromosome in the nuclei demonstrated unambiguously that ectopic muscle fibers originated from implanted cells of female donor rats.

By day 7, the transplanted cells formed a vascularized tissue mass consisting of bundles of multinucleated skeletal muscle fibers surrounded by connective tissue. The well-defined alignment and the fusiform appearance of transplanted cells indicate that, following the injection of the cell suspension into the subcutaneous space, rearrangement of satellite cells took place prior to cell 
fusion. These results support the idea that muscle fiber phenotype is determined, at least in part, by intrinsic influences that are not dependent on the environment (20). However, the reorientation of the satellite cells cannot explain the discrepancies observed in the diameter of ectopic muscle fibers. Actually, during embryogenesis, the myoblasts align and fuse synchronously to form primary myotubes, providing a suitable scaffold for secondary myoblast fusion and subsequent formation of a uniform muscle fiber population (21). The lack of this environment in the subcutaneous space may be responsible for the random satellite cell fusion, leading to a higher variation of fiber size.

Another interesting aspect of the transplants reported here was the detection of $\mathrm{AChE}$ and $\mathrm{nAChR}$ clustering, feature proteins from the neuromuscular synapse, indicating a high stage of muscle fiber maturation. Some aspects of nAChR expression in transplanted tissue resembled those observed during the formation of the neuromuscular synapse. From day 3 to day 14, the ectopic fibers expressed high-density nAChR aggregates. As aneural cultured muscle fibers, by day 3 , the ectopic fibers expressed multiple plaque-shaped nAChR clusters, whereas by days 7 and 14, the distribution of nAChRs was similar to that observed in adult innervated muscle. Although we showed the existence of peripheral nerves in close proximity to ectopic muscle fibers, we cannot assure that the developed fibers were innervated by motor neurons. Besides, the innervation of muscle fibers originated from ectopic or intramuscular injection of myogenic progenitor cells has only been reported after 4 weeks of implantation $(17,22)$. More re- cently, an elegant study has demonstrated the formation of pretzel-shaped aggregates of nAChRs on muscle fibers by nerve-independent mechanisms. According to Kummer et al. (23), both laminin and fibronectin are able to induce the rearrangement of $\mathrm{nAChR}$ aggregates in aneural-cultured muscle fibers, resembling those found in the adult neuromuscular junction. Since subcutaneous space is an environment rich in laminin, fibronectin and other adhesion molecules (24), it is possible that these molecules contribute to the correct arrangement of nAChR clusters at the ectopic site.

Our results show that, when transplanted into the subcutaneous space, satellite cells obtained from neonatal 2BAW rats are able to differentiate into multinucleated muscle fibers containing $\mathrm{AChE}$ and nAChR-rich domains, similar to those seen in adult skeletal muscle fibers. Since multipotent satellite cells can easily grow, fuse and differentiate into skeletal muscle fibers, the subcutaneous transplantation of freshly isolated satellite cells becomes an accessible approach to study the molecular mechanisms involved in the myogenesis and differentiation of muscle-derived stem cells in vivo. In addition, our results show histocompatibility of rats from the Wistar 2BAW colony. Although isogenicity of the Wistar 2BAW colony, an animal model used by many Brazilian researchers, has not been proved definitively, the recipient rats did not reject the myogenic allografts for up to 14 days, indicating that the 2BAW lineage became homogeneous during the uninterrupted inbreeding of common ancestor rats for more than 5 decades. These data also support the use of this rat lineage in future allograft studies.

\section{References}

1. Sanes JR \& Lichtman JW (1999). Development of the vertebrate neuromuscular junction. Annual Review of Neuroscience, 22: 389442.
2. Perry RL \& Rudnick MA (2000). Molecular mechanisms regulating myogenic determination and differentiation. Frontiers in Bioscience, 5: D750-D767. 
3. Asakura A (2003). Stem cells in adult skeletal muscle. Trends in Cardiovascular Medicine, 13: 123-128.

4. Deasy BM, Jankowski RJ \& Huard J (2001). Muscle-derived stem cells: characterization and potential for cell-mediated therapy. Blood Cells, Molecules, and Diseases, 27: 924-933.

5. Partridge TA, Grounds M \& Sloper JC (1978). Evidence of fusion between host and donor myoblasts in skeletal muscle grafts. $\mathrm{Na}$ ture, 273: 306-308.

6. Snow MH (1978). An autoradiographic study of satellite cell differentiation into regenerating myotubes following transplantation of muscles in young rats. Cell and Tissue Research, 186: 535-540.

7. Taylor DA (2001). Cellular cardiomyoplasty with autologous skeletal myoblasts for ischemic heart disease and heart failure. Current Controlled Trials in Cardiovascular Medicine, 2: 208-210.

8. Taylor DA, Atkins BZ, Hungspreugs P, Jones TR, Reedy MC, Hutcheson KA, Glower DD \& Kraus WE (1998). Regenerating functional myocardium: improved performance after skeletal myoblast transplantation. Nature Medicine, 4: 929-933.

9. Pouzet B, Vilquin JT, Hagege AA, Scorsin M, Messas E, Fiszman M, Schwartz K \& Menasche P (2000). Intramyocardial transplantation of autologous myoblasts: can tissue processing be optimized? Circulation, 102: 210-III-215-III.

10. Leobon B, Garcin I, Menasche P, Vilquin JT, Audinat E \& Charpak S (2003). Myoblasts transplanted into rat infarcted myocardium are functionally isolated from their host. Proceedings of the National Academy of Sciences, USA, 100: 7808-7811.

11. Dorfman J, Duong M, Zibaitis A, Pelletier MP, Shum-Tim D, Li C \& Chiu RC (1998). Myocardial tissue engineering with autologous myoblast implantation. Journal of Thoracic and Cardiovascular Surgery, 116: 744-751.

12. Seale $P$, Asakura A \& Rudnicki MA (2001). The potential of muscle stem cells. Developmental Cell, 1: 333-342.

13. da Costa VL, Lapa AJ \& Godinho RO (2001). Short- and long-term influences of calcitonin gene-related peptide on the synthesis of acetylcholinesterase in mammalian myotubes. British Journal of Pharmacology, 133: 229-236.

14. Godinho RO \& Costa-Jr VL (2003). Regulation of intracellular cyclic AMP in skeletal muscle cells involves the efflux of cyclic nucleotide to the extracellular compartment. British Journal of Pharmacology, 138: 995-1003.

15. Karnovsky MJ \& Roots L (1961). Direct-coloring thiocholine method for cholinesterases. Journal of Histochemistry and Cytochemistry, 12: 219-220.

16. Mirra SS, Hart MN \& Terry RD (1993). Making the diagnosis of Alzheimer's disease. A primer for practicing pathologists. Archives of Pathology and Laboratory Medicine, 117: 132-144.

17. Irintchev A, Rosenblatt JD, Cullen MJ, Zweyer M \& Wernig A (1998). Ectopic skeletal muscles derived from myoblasts implanted under the skin. Journal of Cell Science, 111 (Part 22): 3287-3297.

18. Valle JR (1949). Colônia de ratos 2BAW. Ciência e Cultura, 1: 156.

19. Machida S, Spangenburg EE \& Booth FW (2004). Primary rat muscle progenitor cells have decreased proliferation and myotube formation during passages. Cell Proliferation, 37: 267-277.

20. Stockdale FE (1992). Myogenic cell lineages. Developmental Biology, 154: 284-298.

21. Ontell M \& Kozeka K (1984). Organogenesis of the mouse extensor digitorum longus muscle: a quantitative study. American Journal of Anatomy, 171: 149-161.

22. Pin CL \& Merrifield PA (1997). Developmental potential of rat L6 myoblasts in vivo following injection into regenerating muscles. Developmental Biology, 188: 147-166.

23. Kummer TT, Misgeld T, Lichtman JW \& Sanes JR (2004). Nerveindependent formation of a topologically complex postsynaptic apparatus. Journal of Cell Biology, 164: 1077-1087.

24. Aumailley M \& Rousselle P (1999). Laminins of the dermo-epidermal junction. Matrix Biology, 18: 19-28. 